\title{
Finite element model updating using FRF measurements
}

\author{
Farid Asma* and Amar Bouazzouni \\ Laboratory of Mechanics, Structures and Energetic, University Mouloud Mammeri, LP 17 RP 15000 Tizi-ouzou, \\ Algeria
}

Received 22 November 2004

Revised 22 February 2005

\begin{abstract}
This study presents a new updating method based on measured frequency response functions. The objective function of the minimization procedure is formed by the difference between the measured and the analytical frequency responses. The updating parameters are the correction coefficients related to each the elementary mass and stiffness matrices. While making use of a number of incomplete measurements for some frequencies, one builds a non-linear system of equations. The linearisation of the numerical system leads to an iterative procedure. An intrinsic frequency parametrization is proposed in order to accelerate the convergence of the iterative system. The obtained results are comparable with those of the known least squares methods.
\end{abstract}

\section{Nomenclature}

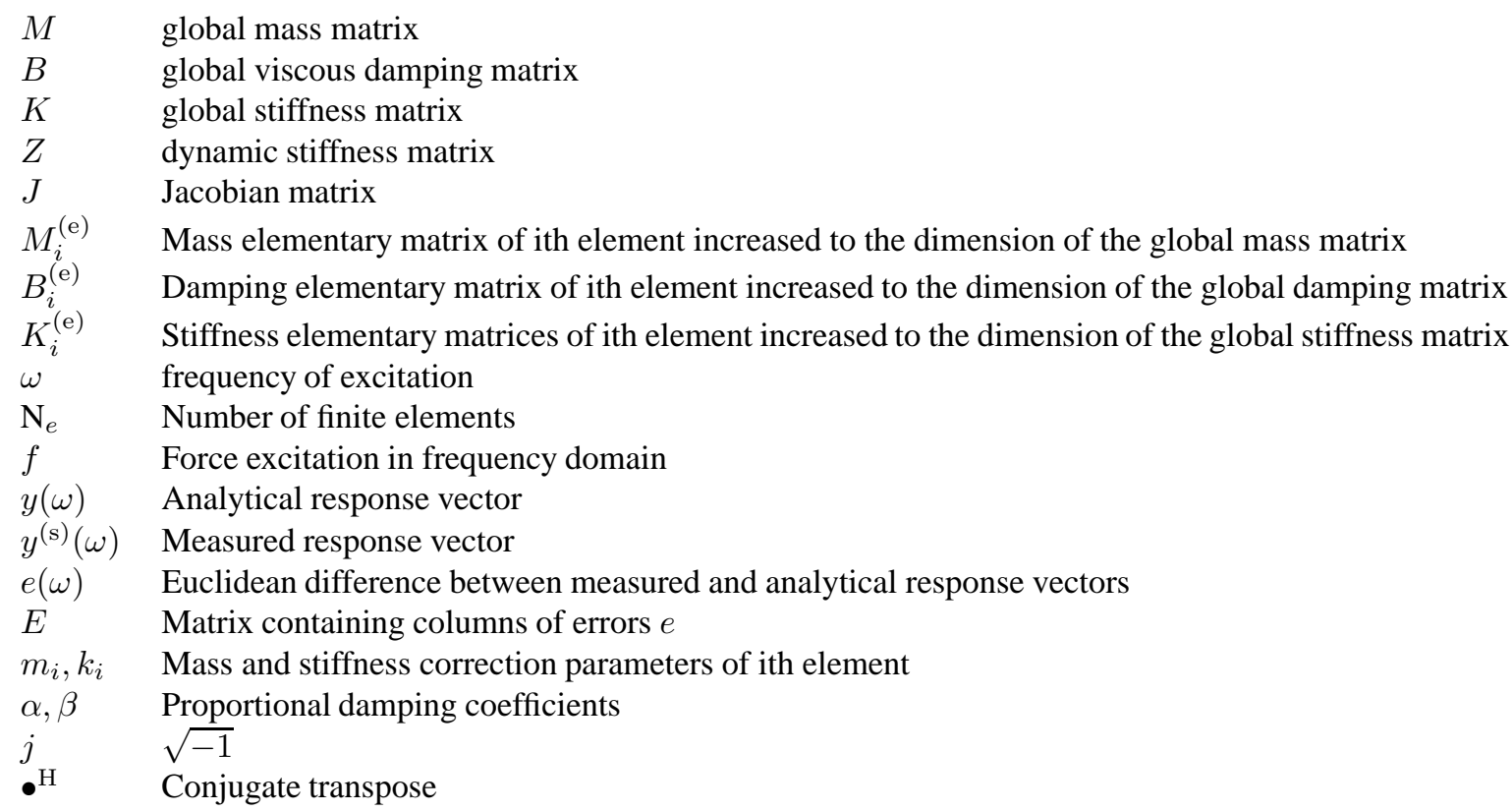




\section{Introduction}

The subject of model updating, in which an initial theoretical model constructed for analyzing the dynamics of structure can be refined, corrected or updated, using test measured on the actual structure, has become one of the most demanding and demanded applications for modal testing [4]. In the case of finite element model updating several number of methods exists and can be classified into four types:

- Direct finite element model updating methods;

- Iterative finite element model updating methods;

- Minimization of modal residuals;

- Minimization of response residuals.

FE model modal based updating algorithms use measured frequency response functions indirectly and rely on experimental modal analysis results, a process which inherently introduces errors and inaccuracies over those already present in the measured data. The use of a limited number of modes is an other source of error. Thus, frequency response function (FRF) model updating methods [1,3,6-9,11,15] are preferable.

In this study, a FRF updating method based on the least squares approximation theory is proposed. The use of FRF measurements avoids us the modal analysis errors.

To ensure fast convergence and improve the stability of the solution, an intrinsic frequency parametrization is proposed.

\section{Equations of motion}

The dynamic behavior of a linear mechanical structure is:

$$
M \ddot{y}(t)+B \dot{y}(t)+K y(t)=f(t)
$$

where the matrices $M, B$ and $K$ are real symmetrical; let these be discretized as follow:

$$
M=\sum_{i=1}^{\mathrm{Ne}} M_{i}^{(\mathrm{e})} B=\sum_{i=1}^{\mathrm{Ne}} B_{i}^{(\mathrm{e})} \text { and } K=\sum_{i=1}^{\mathrm{Ne}} K_{i}^{(\mathrm{e})}
$$

For an harmonic excitation of pulsation $\omega$, the particular solution of Eq. (1) is :

$$
\left(-M \omega^{2}+j \omega \mathrm{B}+\mathrm{K}\right) y=f
$$

from which we can deduce the expression of the response vector:

$$
y(\omega)=\left(-M \omega^{2}+j \omega B+K\right)^{-1} f \text { with } \omega \in\left\{\omega_{1}, \omega_{2}, \ldots, \omega_{S}\right\}
$$

In the implementation of the method, we used a single excitation force. In the following the frequency response function (FRF) is used instead of response vector. However, the method works for the case of multi-excitations.

Damping is considered to be proportional:

$$
B=\alpha M+\beta K
$$

Introducing the parametrization of the structure, the global mass and stiffness matrices may be written as [15]:

$$
M^{(\mathrm{s})}=\sum_{i=1}^{\mathrm{Ne}} m_{i} M_{i}^{(\mathrm{e})} \text { and } K^{(\mathrm{s})}=\sum_{i=1}^{\mathrm{Ne}} k_{i} K_{i}^{(\mathrm{e})}
$$

The case where $m_{i}=k_{i}=1$ corresponds to well modelized ith element.

Let $y^{(\mathrm{s})}\left(\omega_{t}\right)$ be the set of $\mathrm{s}$ incomplete measured response function.

The difference between measured and analytical FRF's can be written [1]:

$$
e\left(\omega_{t}\right)=y\left(\omega_{t}\right)-y^{(\mathrm{s})}\left(\omega_{t}\right)
$$

In practice, the frequency response functions are not completely measured. This issue could be fronted either expanding the experimental data to the analytical ones or reducing the analytical model to the experimental measurements. Although the first approach preserves the physical connections of the whole structure, experience has shown that the latter is generally more successful [2], effect of this limitation is presented by Balis Crema and Coppotelli [2] and Pareira et al. [12]. In the proposed method, the unmeasured degrees of freedom are approximated by their analytical counterpart. 


\section{Approximation method}

The residual error to be minimized for updating purpose is a subject of extensive research in recent years, the success rate of these methods is largely dependent on the optimal choice. The error residual that was minimized by Dascotte and Strobbe [3] is derived from the correlation between all available analytical and experimental FRFs expressed in terms of shape correlation and amplitude correlation.

The well known objective function of the least squares minimization method is:

$$
\delta=\operatorname{Trace}\left(E^{\mathrm{H}} E\right)=\sum_{t=1}^{s} e^{\mathrm{H}}\left(\omega_{t}\right) \cdot e\left(\omega_{t}\right)
$$

The solutions of this are given by:

$$
\frac{\partial \delta}{\partial m_{i}}=0 \text { and } \frac{\partial \delta}{\partial k_{i}}=0(i=1, \ldots, \mathrm{Ne})
$$

This leads to a system with $2 \mathrm{Ne}$ unknowns with $2 \mathrm{Ne}$ equations (see Appendix A).

$$
\left\{\begin{array}{l}
\sum_{t=1}^{s}\left[\left(-\omega_{t}^{2}+j \alpha \omega_{t}\right)\left[e\left(\omega_{t}\right)^{\mathrm{H}}\left(Z^{-1}\left(\omega_{t}\right) \cdot M_{i}^{(\mathrm{e})} \cdot y\left(\omega_{t}\right)\right)+\left(Z^{-1}\left(\omega_{t}\right) \cdot M_{i}^{(\mathrm{e})} \cdot y\left(\omega_{t}\right)\right)^{\mathrm{H}} e\left(\omega_{t}\right)\right]\right]=0 \\
\sum_{t=1}^{s}\left[\left(1+j \beta \omega_{t}\right)\left[e\left(\omega_{t}\right)^{\mathrm{H}}\left(Z^{-1}\left(\omega_{t}\right) \cdot K_{i}^{(\mathrm{e})} \cdot y\left(\omega_{t}\right)\right)+\left(Z^{-1}\left(\omega_{t}\right) \cdot K_{i}^{(\mathrm{e})} \cdot y\left(\omega_{t}\right)\right)^{\mathrm{H}} e\left(\omega_{t}\right)\right]\right]=0 \\
i=1, \ldots, \mathrm{Ne}
\end{array}\right.
$$

The Newton-Raphson resolution leads to an iterative system of the form:

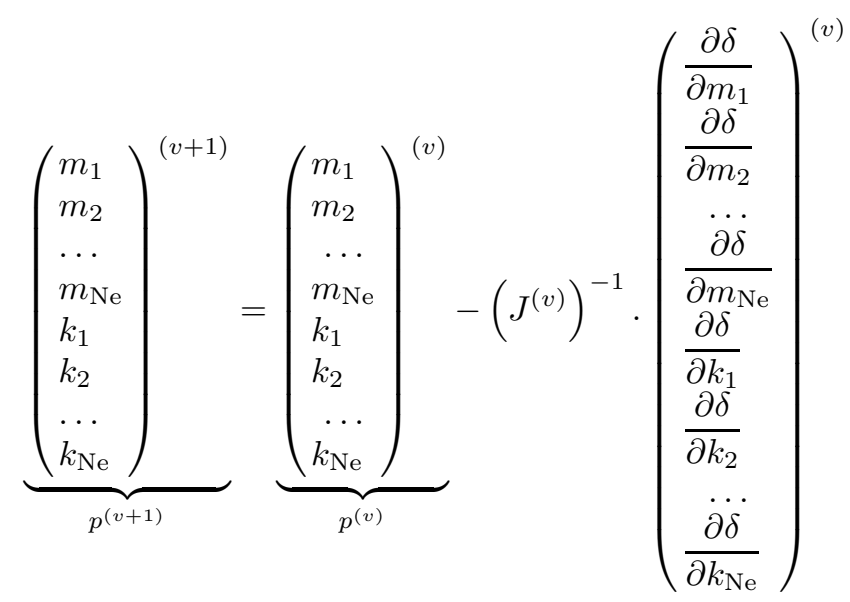

where $p^{(0)}$ is the initial estimation and $J$ is the Jacobian matrix defined by:

- (v) representing the iteration step $v$.

The Jacobian matrix in Eq. (7) is given in Appendix B.

\section{Frequency points measurements}

It is necessary to note that the number and the positioning of the measurement points have an effect on the speed of convergence and the exactitude of the final solution [15].

A more significant number of measurement point improve convergence of the system, but this, can induce a more significant number of iterations for convergence when the points measurement are not in the damaged area (in the area of the elements badly modelized). The measured points play an important role on the model updating procedure. If they do not contain sufficient information about the updated parameters, the procedure will probably not improve 


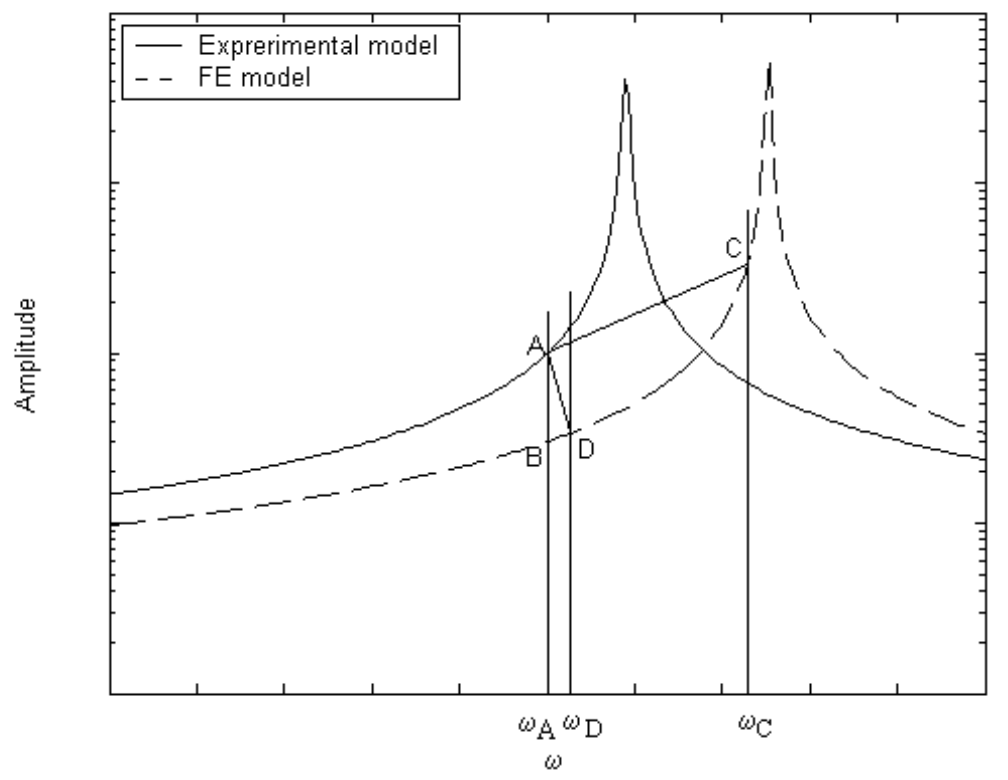

Fig. 1. Different error measurements.

the model correlation [12]. The frequency measurements must be selected away from the resonance to avoid an ill-conditioning of the $\mathrm{J}$ matrix, which could significantly affect convergence. In the case of insufficient number of point measurements, the proposed parametrization may help the iterative updating procedure for convergence. That may also reduce noise effect.

For an updating reliability, it is necessary to envisage different sets of measurements and compare the results obtained to retain only updating parameters having a greater appearance, Trendafilova and Heylen [14] considered three different sets of measurement points, and concluded that precision of the result is determined by the number of the measurement points and their distance to the damage (to the element badly modelized in our case).

\section{Reduction to stiffness updating parameters}

In the case of structural damage, only stiffness matrix is to be corrected, as mass matrix is assumed well modelized. The unknown parameters vector is reduced to those of stiffness $k_{i}$.

The system of Eq. (6) is reduced to:

$$
\sum_{t=1}^{s}\left[\left(1+j \beta \omega_{t}\right)\left[e\left(\omega_{t}\right)^{\mathrm{H}}\left(Z^{-1}\left(\omega_{t}\right) \cdot K_{i}^{(\mathrm{e})} \cdot y\left(\omega_{t}\right)\right)+\left(Z^{-1}\left(\omega_{t}\right) \cdot K_{i}^{(\mathrm{e})} \cdot y\left(\omega_{t}\right)\right)^{\mathrm{H}} e\left(\omega_{t}\right)\right]\right]=0
$$

with $i=1, \ldots, N_{e}$

The Newton-Raphson resolution leads to an iterative system of the form:

$$
\underbrace{\left(\begin{array}{c}
k_{1} \\
k_{2} \\
\vdots \\
k_{\mathrm{Ne}}
\end{array}\right)^{(v+1)}}_{k^{(v+1)}}=\underbrace{\left(\begin{array}{c}
k_{1} \\
k_{2} \\
\vdots \\
k_{\mathrm{Ne}}
\end{array}\right)^{(v)}}_{k^{(v)}}-\left(J^{(v)}\right)^{-1} \cdot\left(\begin{array}{c}
\partial \delta \\
\partial k_{1} \\
\partial \delta \\
\partial k_{2} \\
\vdots \\
\partial \delta \\
\partial k_{\mathrm{Ne}}
\end{array}\right)^{(v)} i=1, \ldots, \mathrm{Ne}
$$




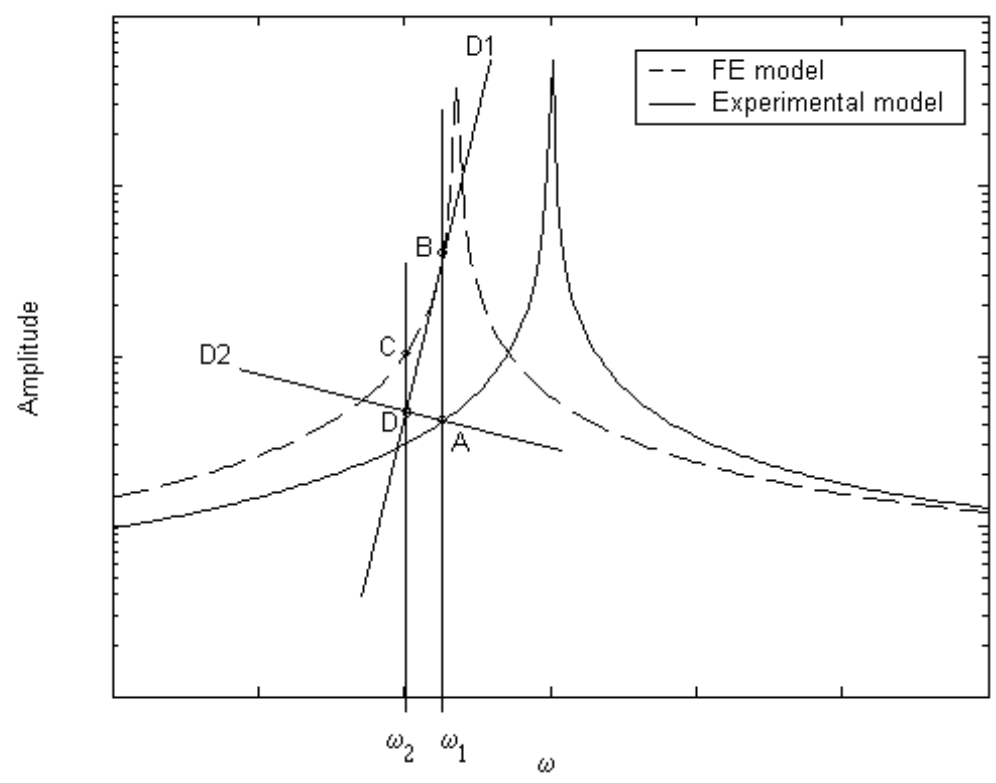

Fig. 2. Geometrical interpretation of the frequency parametrization.

where $J$ is the Jacobian matrix reduced to $J=B$ (Appendix B) and $k^{(0)}$ is the initial estimation of the stiffness updating parameter.

\section{Frequency parametrization}

All methods use residues between experimental and analytical operating deflection shapes at the same frequency(ies) [1]. Pascual et al. [10] introduced the Frequency Domain Assurance Criterion defined as:

$$
\operatorname{FDAC}\left(\omega_{a}, \omega_{x}, j\right)=\begin{gathered}
\left(\left\{H_{a}\left(\omega_{a}\right)\right\}_{j}^{\mathrm{H}}\left\{H_{x}\left(\omega_{x}\right)\right\}_{j}\right)^{2} \\
\left(\left\{H_{a}\left(\omega_{a}\right)\right\}_{j}^{\mathrm{H}}\left\{H_{a}\left(\omega_{a}\right)\right\}_{j}\right)\left(\left\{H_{x}\left(\omega_{x}\right)\right\}_{j}^{\mathrm{H}}\left\{H_{x}\left(\omega_{x}\right)\right\}_{j}\right)
\end{gathered}
$$

where

$j$ corresponds to measured column of $\mathrm{H}$;

$\omega_{a}$ corresponds to the frequency at which $\left\{H_{a}\right\}_{j}$ is calculated;

$\omega_{x}$ corresponds to one frequency at which the FRF was measured experimentally.

This can be regarded as equivalent to the Modal Assurance Criterion [5] in the frequency domain.

Based on the FDAC, Pascual et al. [11] proposed to measure the closeness between measured and synthetized operating deflection shapes by using $\omega_{i}^{j}$ representing the frequency at which FDAC reaches its maximum.

In all cases we do not minimize the shortest distances between the given measured points and the FE approximation. We obtain a situation as described in Fig. 1, where:

A: measured points at frequency $\omega_{A}$;

B: analytical counterpart of $\mathrm{A}$ at the same frequency value;

C: FE frequency calculated point at $\omega_{C}$ which maximizes FDAC;

D: FE frequency calculated point at $\omega_{D}$ according to the normal to the analytical response.

$\mathrm{AD}$ representing the orthogonal error is the shortest distance between the analytical and measured FRFs which may be minimized. This distance is approximated by the projection on the tangent $\mathrm{D}_{2}$ (linear approximation of $y(\omega)$ 


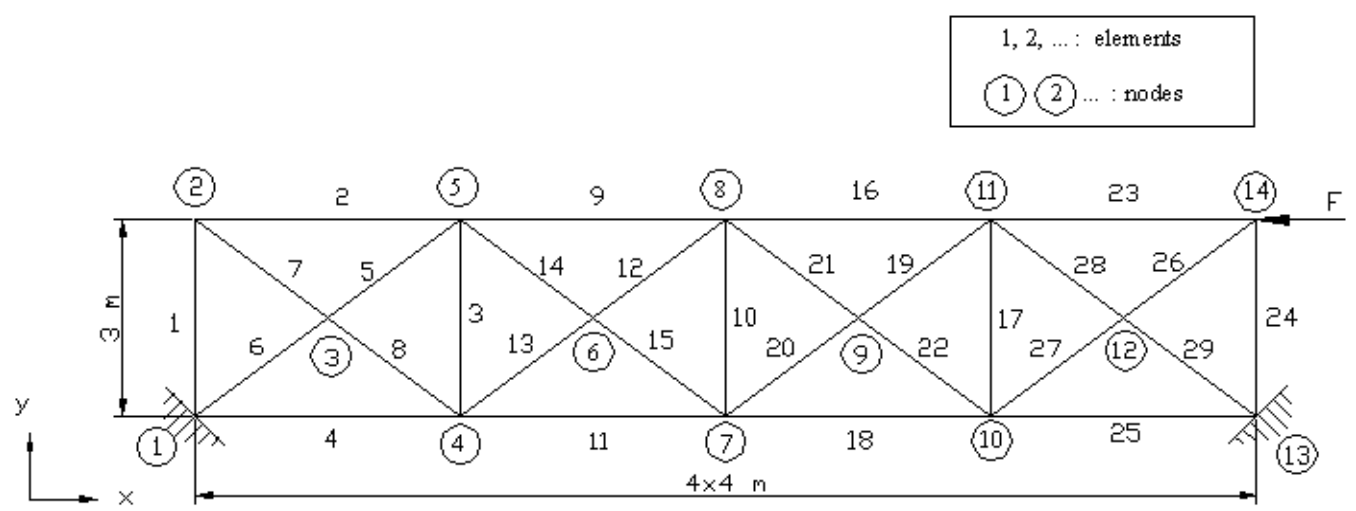

Fig. 3. Structure out of lattice doubly embedded.

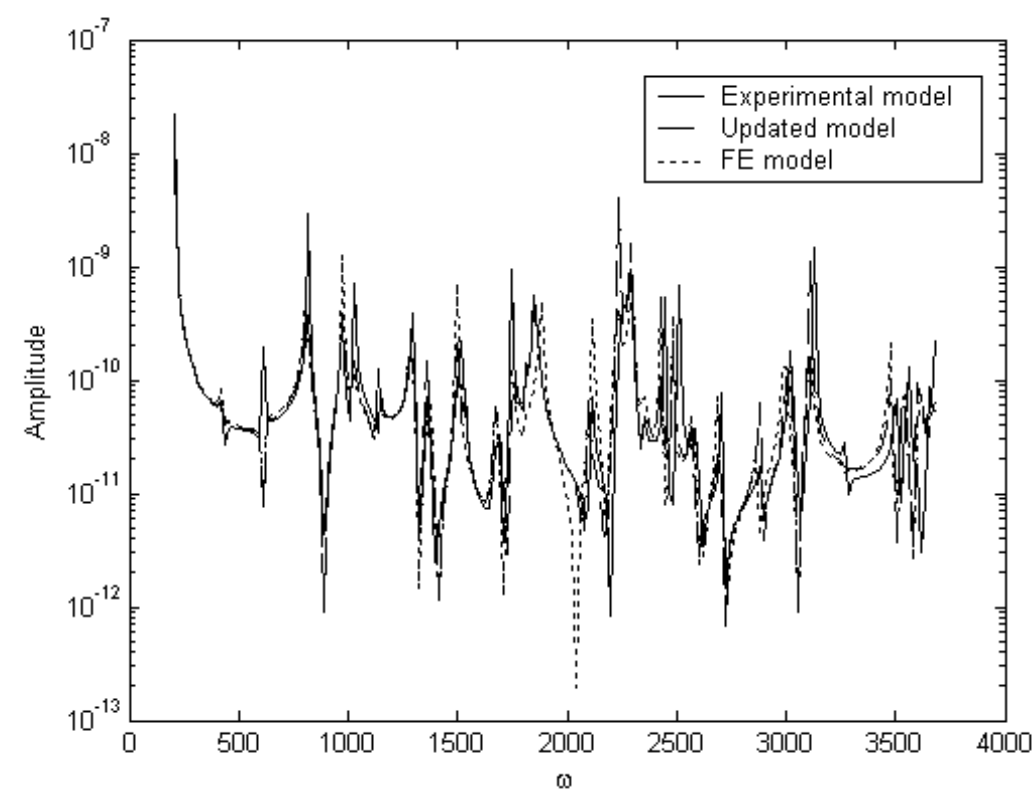

Fig. 4. Response functions comparison of the 20 dof.

in the neighborhood of $\left.y\left(\omega_{i}\right)\right)$ and obtain the changing value $\omega 2$ (Fig. 2), parameter value of the perpendicular from the frequency point measured $A$ to the tangent $\mathrm{D}_{1}$.

Instead of considering the distance between calculated and measured curves for the same frequency (points A and B of Fig. 2), we use the difference between these curves according to their normal (points A and C Fig. 2); the precision of obtained results is better.

Indeed, the error given by Eq. (3) should be measured along the normal to the analytical response curve; this normal is approximated by the line $\mathrm{D}_{2}$ passing across point $\mathrm{A}$ and perpendicular to line $D_{1}$ representing the tangent at point $\mathrm{B}$ to the analytical response curve.

$\omega_{1}$ : measurement frequency value of point $\mathrm{A}, \omega_{t}$;

$\omega_{2}$ : frequency value of point $\mathrm{C}$, intersection of the normal with the structural response function curve passing through A, $\omega_{2 t}$.

Thus, Eq. (3) becomes 
Table 1

Correction results

$\begin{array}{ccc}\text { Element } & \begin{array}{c}\text { Exact perturbation } \\ \text { coefficients }(\%)\end{array} & \begin{array}{c}\text { Identified perturbation } \\ \text { coefficients }(\%)\end{array} \\ 1 & 0 & 0.43 \\ 2 & \mathbf{3 0} & \mathbf{1 3 . 2 5} \\ 3 & 0 & 3.97 \\ 4 & 0 & -5.63 \\ 5 & 0 & 3.97 \\ 6 & 0 & -1.80 \\ 7 & 0 & 0.74 \\ 8 & 0 & -0.47 \\ 9 & 0 & 1.82 \\ 10 & 0 & -1.70 \\ 11 & 0 & -0.90 \\ 12 & \mathbf{2 0} & \mathbf{1 3 . 4 8} \\ 13 & 0 & 1.53 \\ 14 & 0 & 3.09 \\ 15 & 0 & 2.75 \\ 16 & 0 & -0.21 \\ 17 & \mathbf{4 0} & \mathbf{3 8 . 1 3} \\ 18 & 0 & 0.95 \\ 19 & 0 & 4.10 \\ 20 & 0 & -4.04 \\ 21 & 0 & 3.97 \\ 22 & 0 & 2.91 \\ 23 & 0 & 2.53 \\ 24 & 0 & 0.3 \\ 25 & \mathbf{2 0} & 0.25 \\ 26 & 0 & -2.45 \\ 27 & 0 & 0.29 \\ 28 & 0 & 7.83 \\ 29 & 0 & \end{array}$

$$
e\left(\omega_{t}\right)=y\left(\omega_{2 t}\right)-y^{(\mathrm{s})}\left(\omega_{t}\right)
$$

For the parametrization, at each iteration one must ensure that point B do not coincide with the analytical frequency resonance.

\section{Noisy simulated data}

It is well known in modal testing that the quality of measured frequency response functions is adversely affected by noise originating from environment as well as electronic devices [13].

The case of noisy measured FRFs was also investigated. In our case, to simulate measurements, random noise is added according to the model [15]:

$$
y^{(\mathrm{s})}\left(i, \omega_{t}\right)=(1+\text { r.d.n } \%) y^{(\mathrm{s})}\left(i, \omega_{t}\right)
$$

where $r$ is equal randomly to 1 or -1 ,

$d$ : random value between 0 and 1 ,

$n$ : noise percentage.

Simulation was led until a rate of $n=5 \%$.

The level of noise in the measured data determines the reliability and the success of the analyses. It is therefore highly desirable to eliminate or minimize these contaminations from measurement FRFs before they are used for further applications [13]. 


\section{Numerical test case}

Let us consider the plane lattice structure doubly embedded made up of 29 welded beams (Fig. 3) forced by a time-harmonic transverse force $\mathrm{F}=1 \mathrm{~N}$ at the node $14 \mathrm{in} x$ direction. The structure is considered made of steel with Young's modulus $E=2 \times 10^{11} \mathrm{~Pa}$ and mass density $\rho=7850 \mathrm{~kg} / \mathrm{m}^{3}$. The beams have a circular cross-section of $25 \mathrm{~cm}$ diameter. The structure is discretized into 29 finite elements with 36 degrees of freedom. To simulate the real structure, defaults of $+30 \%,+20 \%,+40 \%$, and $+20 \%$ to the stiffness are introduced respectively in the elements 2 , 12, 17 and 25 and 5\% of random noise was added. Point measurements are taken along degrees of freedom 5, 8, 17, 20,26 and 29 of the structure for a selected set of frequency values $\{755,1225,1630,1960,2810,2970,3170\} \mathrm{Hz}$.

Figure 4 and Table 1 illustrate the results obtained. The performance of the method is highlighted by the obtained results. Introduced defaults were detected and quantified.

Indeed, it can be seen that the result obtained (curve in indents) coincides with the simulated measured response function (continuous line curve) after correcting the analytical model (dotted line curve). Table 1 also shows that the simulated defects are localized and quantified for noise ratio going up to $5 \%$ with a reduced number of point measurements (6 DOF of vertical displacement are measured).

\section{Conclusion}

A finite element model updating method based on frequency response function measurements is proposed.

The system convergence depends on the choice and the quality of measurements. To have a good conditioning of the $\mathrm{J}$ matrix, it is essential to avoid approaching the resonance peaks in the real structure case and the corrected analytical structure for each iteration; for this, it is essential to determine for each iteration the resonance frequencies of the updated system in order to eliminate measurements close to these values. The method could still be improved by a judicious measurement point selection.

Aided by the suggested frequency parametrization which chooses the shortest distance to be minimized the method developed shows good convergence properties.

\section{References}

[1] W. D'Ambrogio and A. Fregolent, The use of antiresonances for robust model updating, Journal of Sound and Vibration 236(2) (September 2000), 227-243.

[2] L.B. Crema and G. Coppotelli, Effect of limited DOFs and noise in structural updating, IMAC 2002, pp. 1091-1097.

[3] E. Dascotte and J. Strobbe, Updating finite element models using FRF correlation functions, IMAC 1999, pp. 1169-1174.

[4] D.J. Ewins, Adjustment or updating of models, (Vol 25), Sadhana, Part 3, June 2000, pp. 235-245.

[5] D. Fotsch and D.J. Ewins, Application of the MAC in the frequency domain, IMAC 2000, pp. 1225-1231.

[6] H. Grafe, Model updating of large structural dynamics models using measured response functions, Ph.D. Thesis, Imperial College of Science, Technology and Medicine, Department of Mechanical Engineering, London, UK, October, 1998.

[7] S.R. Ibrahim, W. Teichert and O. Brunner, Frequency response function FE model updating using multi perturbed analytical models and information density matrix, IMAC 1998, pp. 1068-1074.

[8] M. Imregun and W.J. Visser, A review of model updating techniques, Shock and Vibration Digest 23(1) (January, 1991), 9-20.

[9] R.M. Lin and D.J. Ewins, Model updating using FRF data, ISMA 1990, 141-162.

[10] R. Pascual, J.C. Golinval and M. Razeto, A frequency domain correlation technique for model correlation and updating, IMAC 1997, 587-592.

[11] R. Pascual, M. Razeto, J.C. Golinval and R. Schalchli, A robust FRF-based technique for model updating, ISMA 2002, $1037-1046$.

[12] J.A. Pereira, W. Heylen, S. Lammens and P. Sas, Influence of the number of frequency points and resonance frequencies on model updating techniques for health condition monitoring and damage detection of flexible structure, IMAC 1995, $1273-1281$.

[13] K.Y. Sanliturk and O. Cakar, A new method for noise elimination from measured frequency response functions, Mechanical Systems and Signal Processing 19(3) (May, 2005), 615-631.

[14] I. Trendafilova and W. Heylen, Fault localization in structures from remote FRF measurements, Influence of the measurement points, ISMA 1998, 149-156.

[15] W.J. Visser, Updating Structural Dynamics Models Using Frequency Response Data, Ph.D. Thesis, Imperial College of Science, Technology \& Medicine, Department of Mechanical Engineering, London, UK, September, 1992. 


\section{Appendix A: Analytical development of the approximation method}

The well known objective function of the least squares minimization method is:

$$
\delta=\operatorname{Trace}\left(E^{\mathrm{H}} E\right)=\sum_{t=1}^{s} e^{\mathrm{H}}\left(\omega_{t}\right) \cdot e\left(\omega_{t}\right)
$$

The solutions of this are given by:

$$
\partial m_{i}=0 \text { and } \begin{gathered}
\partial \delta \\
\partial k_{i}
\end{gathered}=0(i=1, \ldots, \mathrm{Ne})
$$

This leads to a system with $2 \mathrm{Ne}$ unknowns with $2 \mathrm{Ne}$ equations.

Using Eq. (A1), the preceding equations can be rewritten as follows:

$$
\begin{aligned}
& \frac{\partial \delta}{\partial m_{i}}=\underset{\partial m_{i}}{\partial} \sum_{t=1}^{s} e^{\mathrm{H}}\left(\omega_{t}\right) \cdot e\left(\omega_{t}\right) \\
& \frac{\partial \delta}{\partial m_{i}}=\sum_{t=1}^{s} \frac{\partial}{\partial m_{i}}\left(e^{\mathrm{H}}\left(\omega_{t}\right) \cdot e\left(\omega_{t}\right)\right) \\
& \underset{\partial m_{i}}{\partial \delta}=\sum_{t=1}^{s}\left(\begin{array}{c}
\partial e^{\mathrm{H}}\left(\omega_{t}\right) \\
\partial m_{i}
\end{array} \cdot e\left(\omega_{t}\right)+e^{\mathrm{H}}\left(\omega_{t}\right) \cdot \begin{array}{c}
\partial e\left(\omega_{t}\right) \\
\partial m_{i}
\end{array}\right) \\
& { }_{\partial m_{i}}^{\partial \delta}=\sum_{t=1}^{s}\left[\left(e^{\mathrm{H}}\left(\omega_{t}\right) \cdot \begin{array}{c}
\partial e\left(\omega_{t}\right) \\
\partial m_{i}
\end{array}\right)+\left(e^{\mathrm{H}}\left(\omega_{t}\right) \cdot \begin{array}{c}
\partial e\left(\omega_{t}\right) \\
\partial m_{i}
\end{array}\right)^{\mathrm{H}}\right]
\end{aligned}
$$

In the same way, we may obtain:

$$
\begin{gathered}
\partial \delta \\
\partial k_{i}
\end{gathered}=\sum_{t=1}^{s}\left[\left(e^{\mathrm{H}}\left(\omega_{t}\right) \cdot \begin{array}{c}
\partial e\left(\omega_{t}\right) \\
\partial k_{i}
\end{array}\right)+\left(e^{\mathrm{H}}\left(\omega_{t}\right) \cdot \begin{array}{c}
\partial e\left(\omega_{t}\right) \\
\partial k_{i}
\end{array}\right)^{\mathrm{H}}\right]
$$

Equations (A2) become:

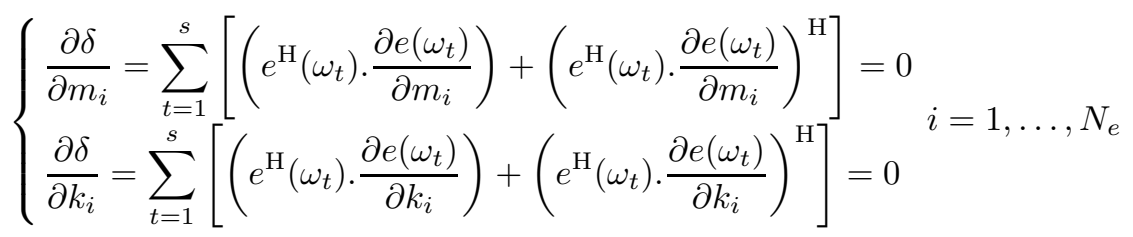

Developing these gives:

$$
\begin{aligned}
& \begin{array}{c}
\partial e\left(\omega_{t}\right) \\
\partial m_{i}
\end{array}=\underset{\partial m_{i}}{\partial}\left[\left(-M \omega_{t}^{2}+j \omega_{t} B+K\right)^{-1} f-y^{(\mathrm{s})}\left(\omega_{t}\right)\right] \\
& \frac{\partial e\left(\omega_{t}\right)}{\partial m_{i}}=\underset{\partial m_{i}}{\partial}\left[Z^{-1}\left(\omega_{t}\right) \cdot f-y^{(\mathrm{s})}\left(\omega_{t}\right)\right] \\
& \begin{array}{c}
\partial e\left(\omega_{t}\right) \\
\partial m_{i}
\end{array}=\begin{array}{c}
\partial Z^{-1}\left(\omega_{t}\right) \\
\partial m_{i}
\end{array} \cdot f
\end{aligned}
$$

Knowing that:

$$
\begin{gathered}
\partial Z^{-1}\left(\omega_{t}\right) \\
\partial m_{i}
\end{gathered}=-Z^{-1}\left(\omega_{t}\right) \cdot \frac{\partial Z\left(\omega_{t}\right)}{\partial m_{i}} \cdot Z^{-1}\left(\omega_{t}\right)
$$


the derivative $Z^{-1}$ can be calculated as follows:

$$
\begin{aligned}
& \begin{array}{c}
\partial Z\left(\omega_{t}\right) \\
\partial m_{i}
\end{array}=\underset{\partial m_{i}}{\partial} \cdot\left(-M \omega_{t}^{2}+j \omega_{t} B+K\right) \\
& \frac{\partial Z\left(\omega_{t}\right)}{\partial m_{i}}=-{ }_{\partial m_{i}} \omega_{t}^{2}+j \omega_{t} \frac{\partial B}{\partial m_{i}}+\begin{array}{c}
\partial K \\
\partial m_{i}
\end{array} \\
& \frac{\partial Z\left(\omega_{t}\right)}{\partial m_{i}}={ }_{\partial m_{i}}^{\partial M}\left(-\omega_{t}^{2}+j \alpha \omega_{t}\right)
\end{aligned}
$$

The derivative of mass matrix M may be derived as:

$$
\frac{\partial M}{\partial m_{i}}=\underset{\partial m_{i}}{\partial}\left(\sum_{v=1}^{\mathrm{Ne}} m_{v} \cdot M_{v}^{(\mathrm{e})}\right)
$$

with

$$
\frac{\partial M}{\partial m_{i}}=M_{i}^{(\mathrm{e})}
$$

Substituting Eq. (A6) into Eq. (A5) one obtains that:

$$
\begin{gathered}
\partial Z\left(\omega_{t}\right) \\
\partial m_{i}
\end{gathered}=M_{i}^{(\mathrm{e})}\left(-\omega_{t}^{2}+j \alpha \omega_{t}\right)
$$

Then substituting this latter into Eq. (A5) leads to:

$$
\begin{gathered}
\partial Z^{-1}\left(\omega_{t}\right) \\
\partial m_{i}
\end{gathered}=-\left(-\omega_{t}^{2}+j \alpha \omega_{t}\right) \cdot Z^{-1}\left(\omega_{t}\right) \cdot M_{i}^{(\mathrm{e})} \cdot Z^{-1}\left(\omega_{t}\right)
$$

Using Eq. (A8) into Eq. (A3) one obtains:

$$
\begin{gathered}
\partial e\left(\omega_{t}\right) \\
\partial m_{i}
\end{gathered}=-\left(-\omega_{t}^{2}+j \alpha \omega_{t}\right) Z^{-1}\left(\omega_{t}\right) \cdot M_{i}^{(\mathrm{e})} \cdot y\left(\omega_{t}\right)
$$

Pre-multiplying by $e^{\mathrm{H}}\left(\omega_{t}\right)$ gives

$$
e^{\mathrm{H}}\left(\omega_{t}\right) \cdot \frac{\partial e\left(\omega_{t}\right)}{\partial m_{i}}=-\left(-\omega_{t}^{2}+j \alpha \omega_{t}\right) e\left(\omega_{t}\right)^{\mathrm{H}} Z^{-1}\left(\omega_{t}\right) \cdot M_{i}^{(\mathrm{e})} \cdot y\left(\omega_{t}\right)
$$

i.e.

$$
{ }_{\partial m_{i}}^{\partial \delta}=-\sum_{t=1}^{s}\left[\left(-\omega_{t}^{2}+j \alpha \omega_{t}\right)\left[e\left(\omega_{t}\right)^{\mathrm{H}}\left(Z^{-1}\left(\omega_{t}\right) \cdot M_{i}^{(\mathrm{e})} \cdot y\left(\omega_{t}\right)\right)+\left(Z^{-1}\left(\omega_{t}\right) \cdot M_{i}^{(\mathrm{e})} \cdot y\left(\omega_{t}\right)\right)^{\mathrm{H}} e\left(\omega_{t}\right)\right]\right]
$$

In the way as was derived Eq. (A7), ones may obtain the following result:

$$
\begin{gathered}
\partial Z\left(\omega_{t}\right) \\
\partial k_{i}
\end{gathered}=K_{i}^{(\mathrm{e})}\left(1+\beta \omega_{t}\right)
$$

Differentiating Eq. (A10), one gets

$$
\begin{gathered}
\partial Z^{-1}\left(\omega_{t}\right) \\
\quad \partial k_{i}
\end{gathered}=-\left(1+j \beta \omega_{t}\right) \cdot Z^{-1}\left(\omega_{t}\right) \cdot K_{i}^{(\mathrm{e})} \cdot Z^{-1}\left(\omega_{t}\right)
$$

In the way as we have derived Eq. (A9) for parameter $m$ we may proceed to obtain equation for parameter $k$ :

$$
\begin{gathered}
\partial e\left(\omega_{t}\right) \\
\partial k_{i}
\end{gathered}=-\left(1+j \beta \omega_{t}\right) Z^{-1}\left(\omega_{t}\right) \cdot K_{i}^{(\mathrm{e})} \cdot y\left(\omega_{t}\right)
$$

Pre multiplying Eq. (A11) by $\mathrm{e}^{\mathrm{H}}\left(\omega_{t}\right)$ one obtains 


$$
e^{\mathrm{H}}\left(\omega_{t}\right) \cdot \underset{\partial e\left(\omega_{t}\right)}{\partial k_{i}}=-\left(1+j \beta \omega_{t}\right) e\left(\omega_{t}\right)^{\mathrm{H}} Z^{-1}\left(\omega_{t}\right) \cdot K_{i}^{(\mathrm{e})} \cdot y\left(\omega_{t}\right)
$$

This leads to

$$
\frac{\partial \delta}{\partial k_{i}}=-\sum_{t=1}^{s}\left[\left(1+j \beta \omega_{t}\right)\left[e\left(\omega_{t}\right)^{\mathrm{H}}\left(Z^{-1}\left(\omega_{t}\right) \cdot K_{i}^{(\mathrm{e})} \cdot y\left(\omega_{t}\right)\right)+\left(Z^{-1}\left(\omega_{t}\right) \cdot K_{i}^{(\mathrm{e})} \cdot y\left(\omega_{t}\right)\right)^{\mathrm{H}} e\left(\omega_{t}\right)\right]\right]
$$

Finally, the following set of equations is obtained:

$$
\left\{\begin{array}{l}
\sum_{t=1}^{s}\left[\left(-\omega_{t}^{2}+j \alpha \omega_{t}\right)\left[e\left(\omega_{t}\right)^{\mathrm{H}}\left(Z^{-1}\left(\omega_{t}\right) \cdot M_{i}^{(\mathrm{e})} \cdot y\left(\omega_{t}\right)\right)+\left(Z^{-1}\left(\omega_{t}\right) \cdot M_{i}^{(\mathrm{e})} \cdot y\left(\omega_{t}\right)\right)^{\mathrm{H}} e\left(\omega_{t}\right)\right]\right]=0 \\
\sum_{t=1}^{s}\left[\left(1+j \beta \omega_{t}\right)\left[e\left(\omega_{t}\right)^{\mathrm{H}}\left(Z^{-1}\left(\omega_{t}\right) \cdot K_{i}^{(\mathrm{e})} \cdot y\left(\omega_{t}\right)\right)+\left(Z^{-1}\left(\omega_{t}\right) \cdot K_{i}^{(\mathrm{e})} \cdot y\left(\omega_{t}\right)\right)^{\mathrm{H}} e\left(\omega_{t}\right)\right]\right]=0
\end{array}\right.
$$

\section{Appendix B: Expression of the Jacobian matrix}

The Jacobian matrix of Eq. (7) is defined as:

$$
J^{(v)}=\left(\begin{array}{cccccccc}
\partial^{2} \delta & \partial^{2} \delta & & \partial^{2} \delta & \partial^{2} \delta & \partial^{2} \delta & & \partial^{2} \delta \\
\partial m_{1}^{2} & \partial m_{2} \partial m_{1} & \cdots & \partial m_{\mathrm{Ne}} \partial m_{1} & \partial k_{1} \partial m_{1} & \partial k_{2} \partial m_{1} & \cdots & \partial k_{\mathrm{Ne}} \partial m_{1} \\
\partial^{2} \delta & \partial^{2} \delta & & \partial^{2} \delta & \partial^{2} \delta & \partial^{2} \delta & & \partial^{2} \delta \\
\partial m_{1} \partial m_{2} & \partial m_{2}^{2} & \cdots & \partial m_{\mathrm{Ne}} \partial m_{2} & \partial k_{1} \partial m_{2} & \partial k_{2} \partial m_{2} & \cdots & \partial k_{\mathrm{Ne}} \partial m_{2} \\
\cdots & \cdots & \cdots & \cdots & \cdots & \cdots & \cdots & \cdots \\
\partial^{2} \delta & \partial^{2} \delta & \cdots & \partial^{2} \delta & \partial^{2} \delta & \partial^{2} \delta & \cdots & \partial^{2} \delta \\
\partial m_{1} \partial m_{\mathrm{Ne}} & \partial m_{2} \partial m_{\mathrm{Ne}} & \cdots & \partial m_{\mathrm{Ne}}^{2} & \partial k_{1} \partial m_{\mathrm{Ne}} & \partial k_{2} \partial m_{\mathrm{Ne}} & \cdots & \partial k_{\mathrm{Ne}} \partial m_{\mathrm{Ne}} \\
\partial^{2} \delta & \partial^{2} \delta & & \partial^{2} \delta & \partial^{2} \delta & \partial^{2} \delta & & \partial^{2} \delta \\
\partial m_{1} \partial k_{1} & \partial m_{2} \partial k_{1} & \cdots & \partial m_{\mathrm{Ne}} \partial k_{1} & \partial k_{1}^{2} & \partial k_{2} \partial k_{1} & \cdots & \partial k_{\mathrm{Ne}} \partial k_{1} \\
\partial^{2} \delta & \partial^{2} \delta & & \partial^{2} \delta & \partial^{2} \delta & \partial^{2} \delta & & \partial^{2} \delta \\
\partial m_{1} \partial k_{2} & \partial m_{2} \partial k_{2} & \cdots & \partial m_{\mathrm{Ne}} \partial k_{2} & \partial k_{1} \partial k_{2} & \partial k_{2}^{2} & \cdots & \partial k_{\mathrm{Ne}} \partial k_{2} \\
\cdots & \cdots & \cdots & \cdots & \cdots & \cdots & \cdots & \cdots \\
\partial^{2} \delta & \partial^{2} \delta & \cdots & \partial^{2} \delta & \partial^{2} \delta & \partial^{2} \delta & \cdots & \partial^{2} \delta \\
\partial m_{1} \partial k_{\mathrm{Ne}} & \partial m_{2} \partial k_{\mathrm{Ne}} & \cdots & \partial m_{\mathrm{Ne}} \partial k_{\mathrm{Ne}} & \partial k_{1} \partial k_{\mathrm{Ne}} & \partial k_{2} \partial k_{\mathrm{Ne}} & \cdots & \partial k_{\mathrm{Ne}}^{2}
\end{array}\right)^{2}
$$

This may be partitioned as follows

$$
J=\left[\begin{array}{cc}
A & C \\
D & B
\end{array}\right] \text { where A, B, C and D are matrices of } C^{\mathrm{Ne} \times \mathrm{Ne}}
$$

calculating the elements of A, B, D and D of the J matrix we obtain:

$$
\begin{gathered}
A_{i j}=\underset{\partial m_{j} \partial m_{i}}{\partial^{2} \delta}=\sum_{t=1}^{s}\left(A_{t}+A_{t}^{\mathrm{H}}\right) \\
B_{i j}=\begin{array}{c}
\partial^{2} \delta \\
\partial k_{j} \partial k_{i}
\end{array}=\sum_{t=1}^{s}\left(B_{t}+B_{t}^{\mathrm{H}}\right) \\
C_{i j}=\frac{\partial^{2} \delta}{\partial k_{j} \partial m_{i}}=\sum_{t=1}^{s}\left(C_{t}+C_{t}^{\mathrm{H}}\right) \\
D_{i j}=\underset{\partial m_{j} \partial k_{i}}{\partial^{2} \delta}=\sum_{t=1}^{s}\left(D_{t}+D_{t}^{\mathrm{H}}\right)
\end{gathered}
$$


with

$$
\begin{aligned}
A_{t}= & \left(-\omega_{t}^{2}+j \alpha \omega_{t}\right)^{2}\left[\left[\left(Z^{-1} M_{j}^{(\mathrm{e})} Z^{-1} M_{i}^{(\mathrm{e})} y\left(\omega_{t}\right)\right)+\left(Z^{-1} M_{i}^{(\mathrm{e})} Z^{-1} M_{j}^{(\mathrm{e})} y\left(\omega_{t}\right)\right)\right]^{\mathrm{H}} e\left(\omega_{t}\right)\right. \\
& \left.+\left(Z^{-1} M_{i}^{(\mathrm{e})} y\left(\omega_{t}\right)\right)^{\mathrm{H}} \cdot\left(Z^{-1} M_{j}^{(\mathrm{e})} y\left(\omega_{t}\right)\right)\right] \\
B_{t}= & \left(1+j \beta \omega_{t}\right)^{2}\left[\left[\left(Z^{-1} K_{j}^{(\mathrm{e})} Z^{-1} K_{i}^{(\mathrm{e})} y\left(\omega_{t}\right)\right)+\left(Z^{-1} K_{i}^{(\mathrm{e})} Z^{-1} K_{j}^{(\mathrm{e})} y\left(\omega_{t}\right)\right)\right]^{\mathrm{H}} e\left(\omega_{t}\right)+\right. \\
& \left.\left(Z^{-1} K_{i}^{(\mathrm{e})} y\left(\omega_{t}\right)\right)^{\mathrm{H}} \cdot\left(Z^{-1} K_{j}^{(\mathrm{e})} y\left(\omega_{t}\right)\right)\right] \\
C_{t}= & \left(-\omega_{t}^{2}+j \alpha \omega_{t}\right)\left(1+j \beta \omega_{t}\right)\left[\left[\left(Z^{-1} K_{j}^{(\mathrm{e})} Z^{-1} M_{i}^{(\mathrm{e})} y\left(\omega_{t}\right)\right)+\left(Z^{-1} M_{i}^{(\mathrm{e})} Z^{-1} K_{j}^{(\mathrm{e})} y\left(\omega_{t}\right)\right)\right]^{\mathrm{H}} e\left(\omega_{t}\right)\right. \\
& \left.+\left(Z^{-1} M_{i}^{(\mathrm{e})} y\left(\omega_{t}\right)\right)^{\mathrm{H}}\left(Z^{-1} K_{j}^{(\mathrm{e})} y\left(\omega_{t}\right)\right)\right] \\
D_{t}= & \left(-\omega_{t}^{2}+j \alpha \omega_{t}\right)\left(1+j \beta \omega_{t}\right)\left[\left[\left(Z^{-1} M_{j}^{(\mathrm{e})} Z^{-1} K_{i}^{(\mathrm{e})} y\left(\omega_{t}\right)\right)+\left(Z^{-1} K_{i}^{(\mathrm{e})} Z^{-1} M_{j}^{(\mathrm{e})} y\left(\omega_{t}\right)\right)\right]^{\mathrm{H}} e\left(\omega_{t}\right)\right. \\
& \left.+\left(Z^{-1} K_{i}^{(\mathrm{e})} y\left(\omega_{t}\right)\right)^{\mathrm{H}}\left(Z^{-1} M_{j}^{(\mathrm{e})} y\left(\omega_{t}\right)\right)\right]
\end{aligned}
$$

Thus, the Jacobian matrix is then totally defined and its expression is substituted in the iterative system Eq. (7). 

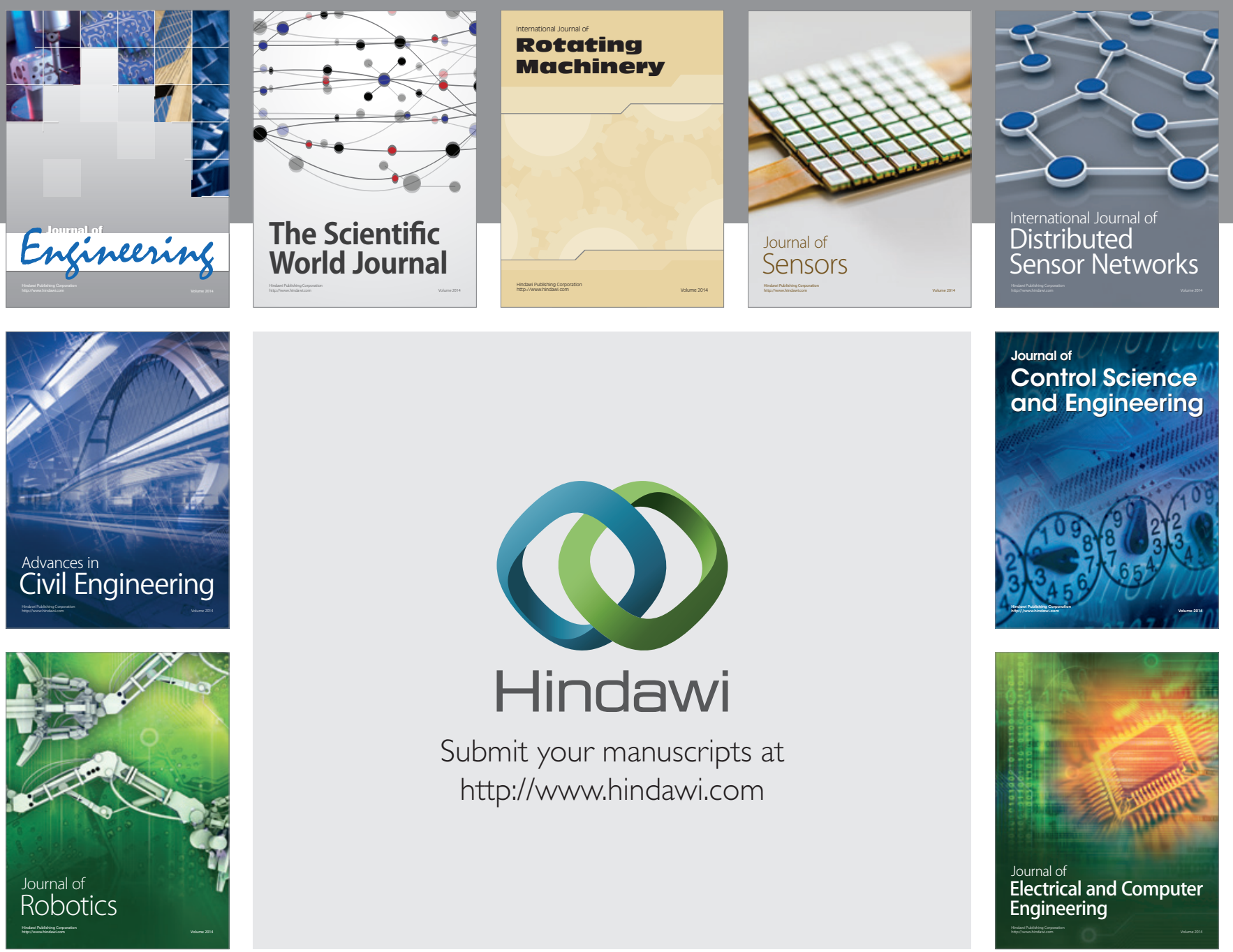

Submit your manuscripts at

http://www.hindawi.com
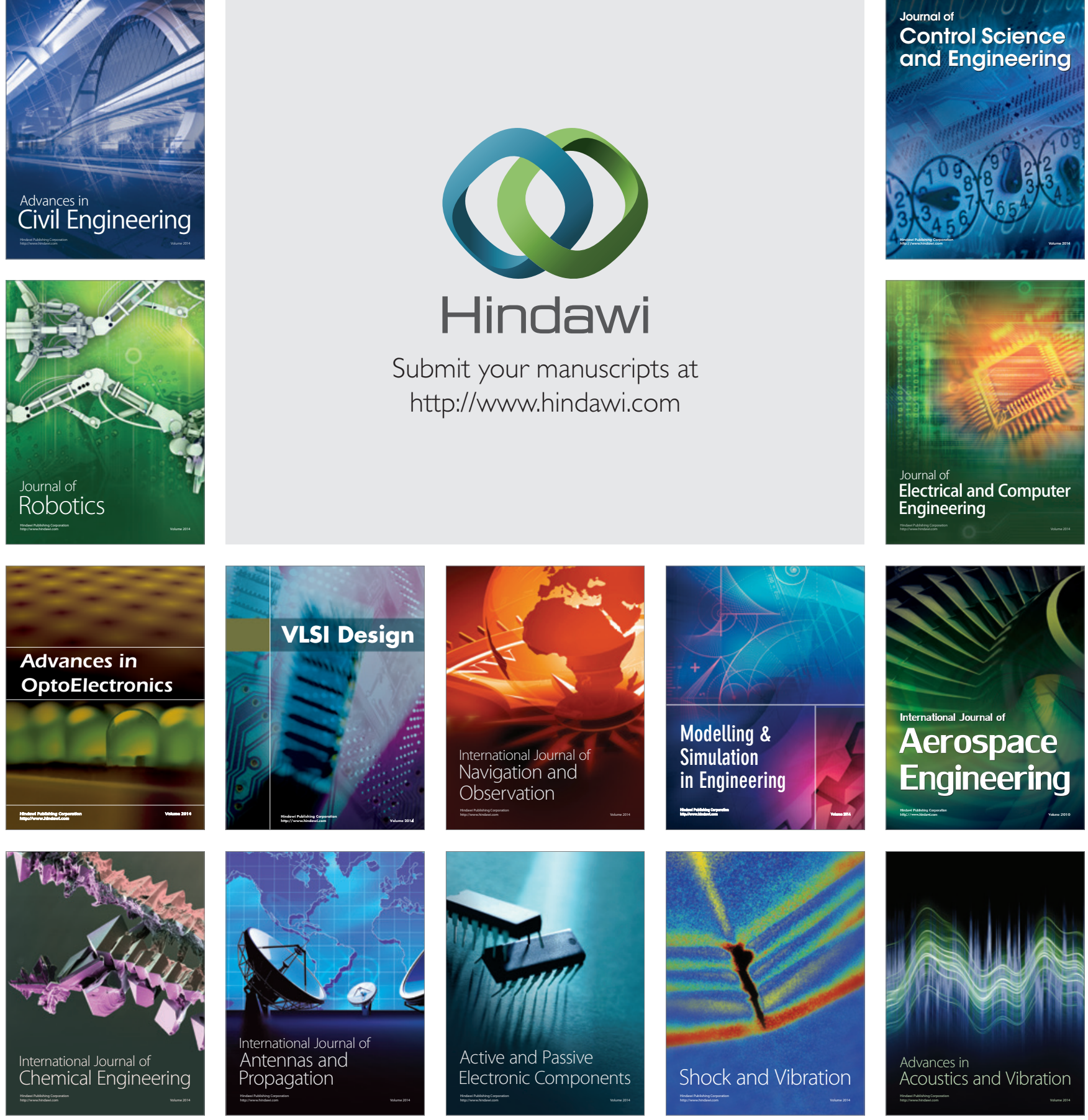